Supplement of Atmos. Chem. Phys. Discuss., 14, 24523-24572, 2014

http://www.atmos-chem-phys-discuss.net/14/24523/2014/

doi:10.5194/acpd-14-24523-2014-supplement

(C) Author(s) 2014. CC Attribution 3.0 License.

(c) (i)

\title{
Receptor modelling of fine particles in Southern England using CMB including comparison with AMS-PMF factors
}

J. Yin et al.

Correspondence to: R. M. Harrison (r.m.harrison@bham.ac.uk) 
Table S1. Listof all organic markers analysed

\begin{tabular}{|c|c|c|c|c|c|}
\hline n-Alkanes & Abbreviation & Hopanes & Abbreviation & PAHs & Abbreviation \\
\hline$n$-Tetracosane & $\mathrm{C} 24$ & $17 \alpha(\mathrm{H})-22,29,30$-Trisnorhopane & $17 \alpha$ TNohop & Benzo[k]fluoranthene & $\mathrm{B}[\mathrm{k}] \mathrm{F}$ \\
\hline$n$-Pentacosane & $\mathrm{C} 25$ & $17 \alpha(\mathrm{H}), 21 \beta(\mathrm{H})$-Hopane & $17 \alpha \beta$ Нор & Benzo[b]fluoranthene & $\mathrm{B}[\mathrm{b}] \mathrm{F}$ \\
\hline$n$-Hexacosane & C26 & $17 \beta(\mathrm{H}), 21 \alpha(\mathrm{H})$-30-norhopane & $17 \beta \alpha$ Nohop & Benzo[e]pyrene & $\mathrm{B}[\mathrm{e}] \mathrm{P}$ \\
\hline n-Heptacosane & C27 & 22S-17 $\alpha(\mathrm{H}), 21 \beta(\mathrm{H})-30$-Homohopane & $22 \mathrm{~S} \alpha \beta \mathrm{HH}$ & Benzo[a]pyrene & $\mathrm{B}[\mathrm{a}] \mathrm{P}$ \\
\hline n-Octacosane & C28 & 22R-17 $\alpha(\mathrm{H}), 21 \beta(\mathrm{H})-30$-Homohopane & $22 \mathrm{R} \alpha \beta \mathrm{HH}$ & Perylene & PER \\
\hline$n$-Nonacosane & C29 & 22S-17 $\alpha(\mathrm{H}), 21 \beta(\mathrm{H})$-30-Bishomohopane & $22 \mathrm{~S} \alpha \beta \mathrm{BHH}$ & Indeno[1,2,3-cd]pyrene & IP \\
\hline$n$-Triacontane & C30 & 22R-17 $\alpha(\mathrm{H}), 21 \beta(\mathrm{H})$-30-Bishomohopane & $22 \mathrm{R} \alpha \beta \mathrm{BHH}$ & Dibenz[a,h]anthracene & $\mathrm{D}[\mathrm{ah}] \mathrm{A}$ \\
\hline n-Hentriacontane & C31 & 22S-17 $\alpha(\mathrm{H}), 21 \beta(\mathrm{H})-30,31,32-$ trishomohopane & $22 \mathrm{~S} \alpha \beta \mathrm{THH}$ & Benzo[ghi]perylene & B[ghi]PER \\
\hline n-Dotriacontane & C32 & 22R-17 $\alpha(\mathrm{H}), 21 \beta(\mathrm{H})-30,31,32$-trishomohopane & 22R $\alpha \beta \mathrm{THH}$ & Coronene & COR \\
\hline n-Tritriacontane & C33 & Fatty acids & & Picene & PIC \\
\hline$n$-Tetratriacontane & C34 & Tetradecanoic acid/Myristic acid (C14) & MyrA & Secondary markers & \\
\hline n-Pentatriacontane & C35 & Pentadecanoic acid/Pentadecylic acid (C15) & PentA & Pinonic acid & PinoA \\
\hline Sterols & & Hexadecanoic acid/Palmitic acid (C16) & PalmA & Pinic acid & PinicA \\
\hline Levoglucosan & Levo & Octadecanoic acid/Stearic acid (C18) & SteaA & 2-methylthreitol & MethT \\
\hline \multirow[t]{2}{*}{ Cholesterol } & Chol & 9-octadecenoic acid/Oleic acid (C18:1) & OleiA & 2-methylerythritol & MethE \\
\hline & & 9,12-octadecadienoic acid/Linoleic acid (C18:2) & LinoA & & \\
\hline
\end{tabular}


Table S2. Precision (\%) and method detection limit (MDL)(ng $\mathrm{m}^{-3}$ ) for the target compounds

\begin{tabular}{|c|c|c|c|c|c|c|c|c|}
\hline n-Alkanes & Precision & MDL & Hopanes & Precision & MDL & PAHS & Precision & MDL \\
\hline $\mathrm{C} 24$ & 5.1 & 0.027 & 17aTNohop & 4.7 & 0.010 & $\mathrm{~B}[\mathrm{~b}] \mathrm{F}$ & 6.2 & 0.015 \\
\hline $\mathrm{C} 25$ & 4.4 & 0.041 & 17abNohop & 5.1 & 0.011 & $\mathrm{~B}[\mathrm{k}] \mathrm{F}$ & 4.3 & 0.017 \\
\hline C26 & 7.1 & 0.055 & 17baNohop & 3.4 & 0.017 & $\mathrm{~B}[\mathrm{e}] \mathrm{P}$ & 5.3 & 0.019 \\
\hline $\mathrm{C} 27$ & 5.7 & 0.026 & 17abНор & 5.5 & 0.014 & $\mathrm{~B}[\mathrm{a}] \mathrm{P}$ & 4.9 & 0.014 \\
\hline C28 & 10.8 & 0.019 & 22SabHH & 4.3 & 0.015 & PER & 5.8 & 0.010 \\
\hline C29 & 13.4 & 0.048 & 22RabHH & 4.4 & 0.018 & IP & 8.9 & 0.008 \\
\hline C30 & 10.7 & 0.066 & 22SabBHH & 5.1 & 0.016 & $\mathrm{D}[\mathrm{ah}] \mathrm{A}$ & 6.6 & 0.011 \\
\hline C31 & 8.1 & 0.034 & 22RabBHH & 5.7 & 0.047 & PIC & 15.0 & 0.025 \\
\hline C32 & 8.8 & 0.044 & 22SabTHH & 5.2 & 0.014 & B[ghi]PER & 7.5 & 0.017 \\
\hline C33 & 15.9 & 0.034 & 22RabTHH & 6.3 & 0.035 & COR & 3.2 & 0.016 \\
\hline C34 & 3.3 & 0.015 & Fatty acids & & & & & \\
\hline C35 & 7.5 & 0.054 & MA & 11.9 & 0.57 & & & \\
\hline Sterols & & & PentA & 8.8 & 0.23 & $\begin{array}{c}\text { Secondary } \\
\text { Markers }\end{array}$ & & \\
\hline Levo & 15.4 & 0.23 & $\mathrm{PA}$ & 20.4 & 2.1 & PinoA & 15.7 & 0.37 \\
\hline \multirow[t]{3}{*}{ Chol } & 5.6 & 0.024 & LOA & 6.8 & 0.097 & PinicA & 8.1 & 0.19 \\
\hline & & & OA & 9.2 & 0.31 & MethT & 5.9 & 0.035 \\
\hline & & & SA & 19.2 & 1.3 & MethE & 7.4 & 0.10 \\
\hline
\end{tabular}


Table S3. Dependence of mean mass of PMF factors upon value of fpeak=0

\begin{tabular}{|c|c|c|c|c|c|}
\hline Factor & $\begin{array}{c}\text { OOA } \\
\text { Mean mass }\end{array}$ & $\begin{array}{c}\text { SFOA1 } \\
\text { Mean mass }\end{array}$ & $\begin{array}{c}\text { SFOA2 } \\
\text { Mean mass }\end{array}$ & $\begin{array}{c}\text { COA } \\
\text { Mean mass }\end{array}$ & $\begin{array}{c}\text { HOA } \\
\text { Mean mass }\end{array}$ \\
\hline-0.6 & 0.897374 & 0.757835 & 0.882626 & 1.02376 & 0.68164 \\
\hline 0 & 0.933063 & 0.747544 & 0.857564 & 0.875335 & 0.829156 \\
\hline 1 & 0.940803 & 0.696752 & 0.776001 & 0.682512 & 1.14724 \\
\hline
\end{tabular}


Table S4: Source contribution estimates (SCE) $\left(\mu \mathrm{g} \mathrm{m}^{-3}\right)$ and standard deviation (S.D.) for fine particulate $\mathrm{OC}$ and $\mathrm{PM}_{2.5}$ at $\mathrm{NK}$ and HAR from the CMB model (averaged from daily CMB outputs)

\begin{tabular}{|c|c|c|c|c|c|c|c|c|}
\hline \multirow{2}{*}{ Source Name } & & \multicolumn{3}{|c|}{ OC } & \multicolumn{3}{|c|}{$\mathbf{P M}_{2.5}$} & \multirow{2}{*}{$\begin{array}{l}\text { OC/PM }_{2.5} \text { or } \\
\text { OC/OM CF }\end{array}$} \\
\hline & & $\mathbf{N K}^{\mathbf{a}}$ & $\mathbf{N K}^{\mathbf{b}}$ & $\mathbf{H A R}^{\mathrm{a}}$ & $\mathbf{N K}^{\mathbf{a}}$ & $\mathbf{N K}^{\mathbf{b}}$ & $\mathbf{H A R}^{\mathbf{a}}$ & \\
\hline \multirow[t]{2}{*}{ Vegetation } & SCE & 0.069 & 0.069 & 0.11 & 0.21 & 0.21 & 0.35 & 0.324 \\
\hline & S.D. & 0.010 & 0.010 & 0.015 & 0.030 & 0.030 & 0.048 & - \\
\hline \multirow[t]{2}{*}{ Woodsmoke } & SCE & 0.53 & 0.53 & 0.64 & 0.64 & 0.64 & 0.77 & 0.836 \\
\hline & S.D. & 0.11 & 0.11 & 0.14 & 0.14 & 0.14 & 0.16 & - \\
\hline \multirow[t]{2}{*}{ Natural Gas } & SCE & 0.046 & 0.046 & 0.042 & 0.054 & 0.054 & 0.050 & 0.849 \\
\hline & S.D. & 0.009 & 0.009 & 0.007 & 0.011 & 0.011 & 0.008 & - \\
\hline \multirow[t]{2}{*}{ Dust/Soil } & SCE & 0.049 & 0.049 & 0.018 & 0.38 & 0.38 & 0.14 & 0.131 \\
\hline & S.D. & 0.037 & 0.037 & 0.015 & 0.29 & 0.29 & 0.11 & 0.0133 \\
\hline \multirow[t]{2}{*}{ Coal } & SCE & 0.075 & 0.075 & 0.041 & 0.17 & 0.17 & 0.094 & 0.432 \\
\hline & S.D. & 0.020 & 0.020 & 0.009 & 0.046 & 0.046 & 0.021 & 0.0834 \\
\hline \multirow[t]{2}{*}{ Food Cooking } & SCE & 0.32 & 0.32 & 0.070 & 0.56 & 0.56 & 0.12 & 0.566 \\
\hline & S.D. & 0.055 & 0.055 & 0.013 & 0.10 & 0.10 & 0.023 & 0.030 \\
\hline \multirow[t]{2}{*}{ Total Traffic } & SCE & 0.81 & 0.81 & 0.35 & 1.40 & 1.40 & 0.59 & 0.579 \\
\hline & S.D. & 0.39 & 0.39 & 0.16 & 0.88 & 0.88 & 0.29 & 0.051 \\
\hline \multirow[t]{2}{*}{ Biogenic Secondary } & $S C E$ & - & 0.90 & - & - & 1.63 & - & 0.556 \\
\hline & S.D. & - & 0.17 & - & - & 0.31 & - & - \\
\hline \multirow[t]{2}{*}{ Other OC/OM } & SCE & 1.62 & 0.72 & 1.03 & 2.92 & 1.29 & 1.85 & 0.556 \\
\hline & S.D. & - & - & - & - & - & - & - \\
\hline \multirow[t]{2}{*}{ Sea Salt } & $S C E$ & - & - & - & 1.1 & 1.1 & 0.82 & - \\
\hline & S.D. & - & - & - & 0.020 & 0.020 & 0.020 & - \\
\hline \multirow{2}{*}{$\begin{array}{c}\text { Ammonium } \\
\text { Sulphate }\end{array}$} & $S C E$ & - & - & - & 2.2 & 2.2 & 2.1 & - \\
\hline & S.D. & - & - & - & 0.028 & 0.028 & 0.028 & - \\
\hline \multirow[t]{2}{*}{ Ammonium Nitrate } & SCE & - & - & - & 5.8 & 5.8 & 4.1 & - \\
\hline & S.D. & - & - & - & 0.072 & 0.072 & 0.072 & - \\
\hline Measured OC/PM 2.5 & Mass & 3.5 & 3.5 & 2.3 & 15.7 & 15.7 & 11.0 & - \\
\hline
\end{tabular}

Note: Figures in bold were not statistically different from zero; $a$ - Modelled without biogenic secondary source profile; $b$ - Modelled with biogenic secondary source profile; $c$ - Conversion factor 


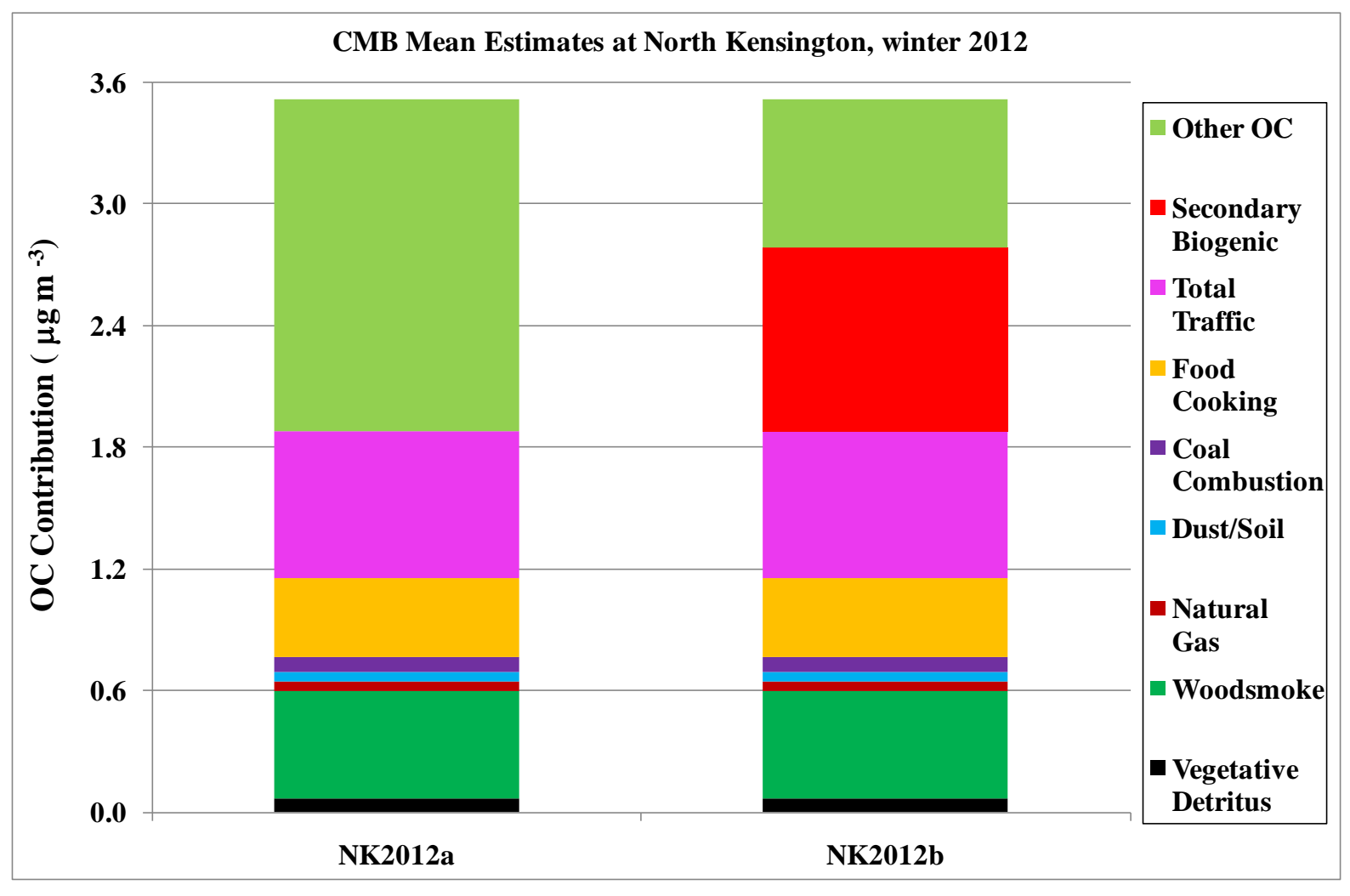

Figure S1: Mean OC source contribution estimates with (b) and without (a) secondary biogenic component at NK 
NOAA HYSPLIT MODEL

Backward trajectory ending at 1200 UTC 13 Jan 12

GDAS Meteorological Data

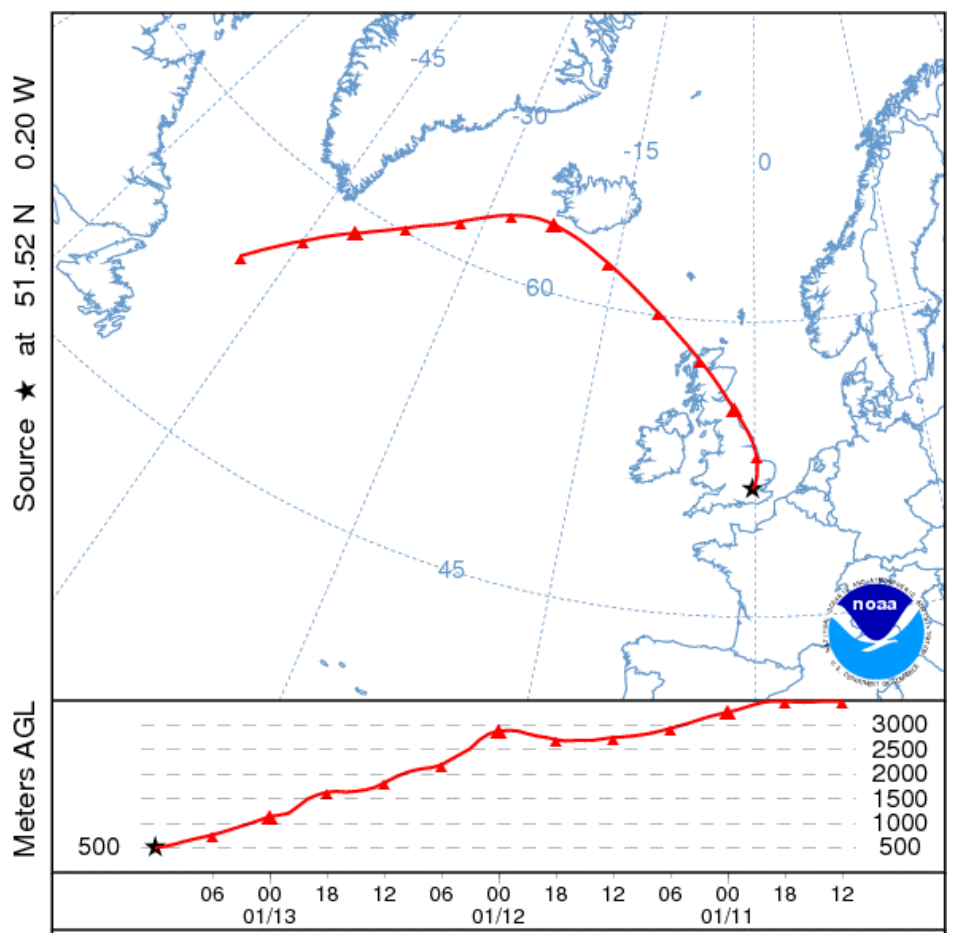

NOAA HYSPLIT MODEL

Backward trajectory ending at 1800 UTC 13 Jan 12 GDAS Meteorological Data

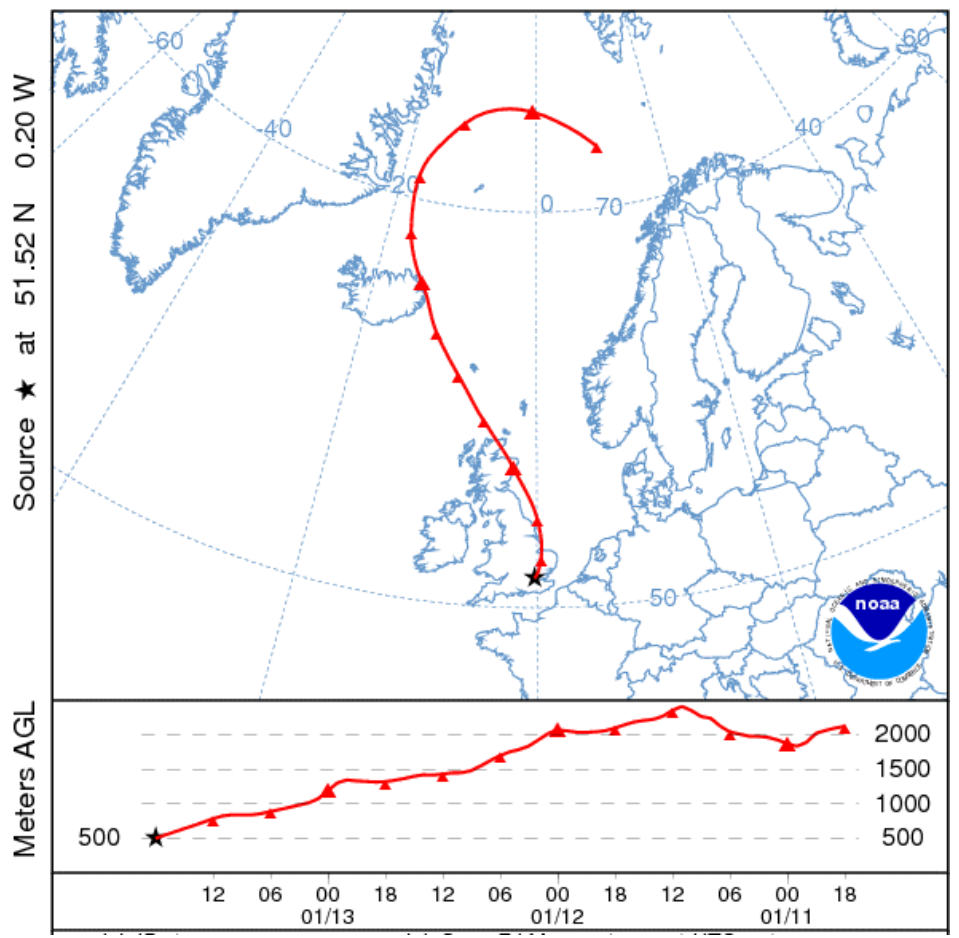


NOAA HYSPLIT MODEL

Backward trajectory ending at 0000 UTC 14 Jan 12

GDAS Meteorological Data

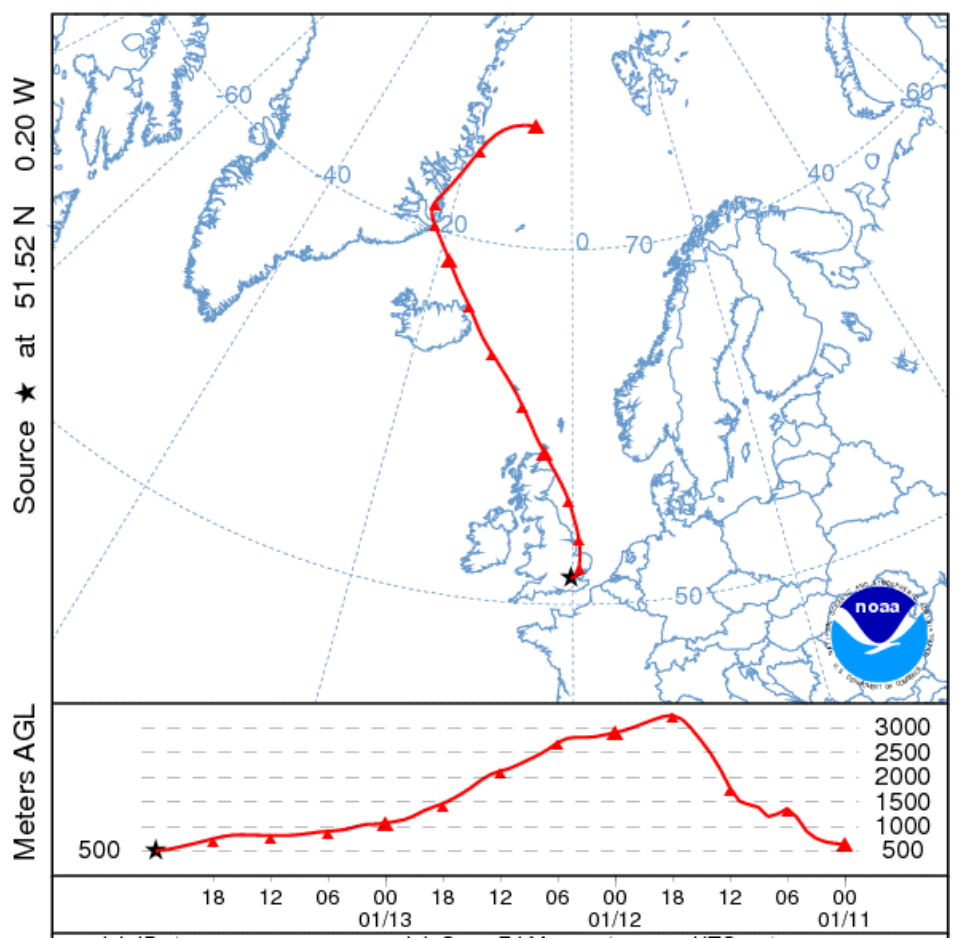

NOAA HYSPLIT MODEL

Backward trajectory ending at 0600 UTC 14 Jan 12 GDAS Meteorological Data

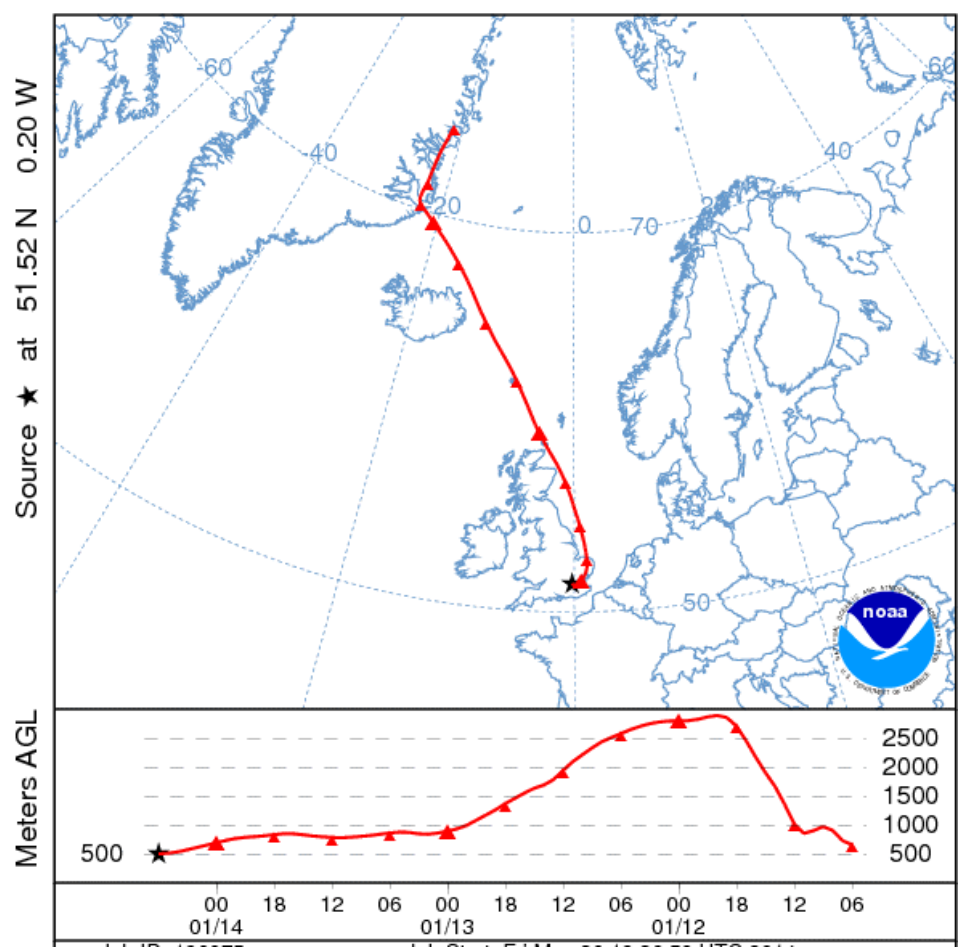


NOAA HYSPLIT MODEL

Backward trajectory ending at 1200 UTC 14 Jan 12

GDAS Meteorological Data

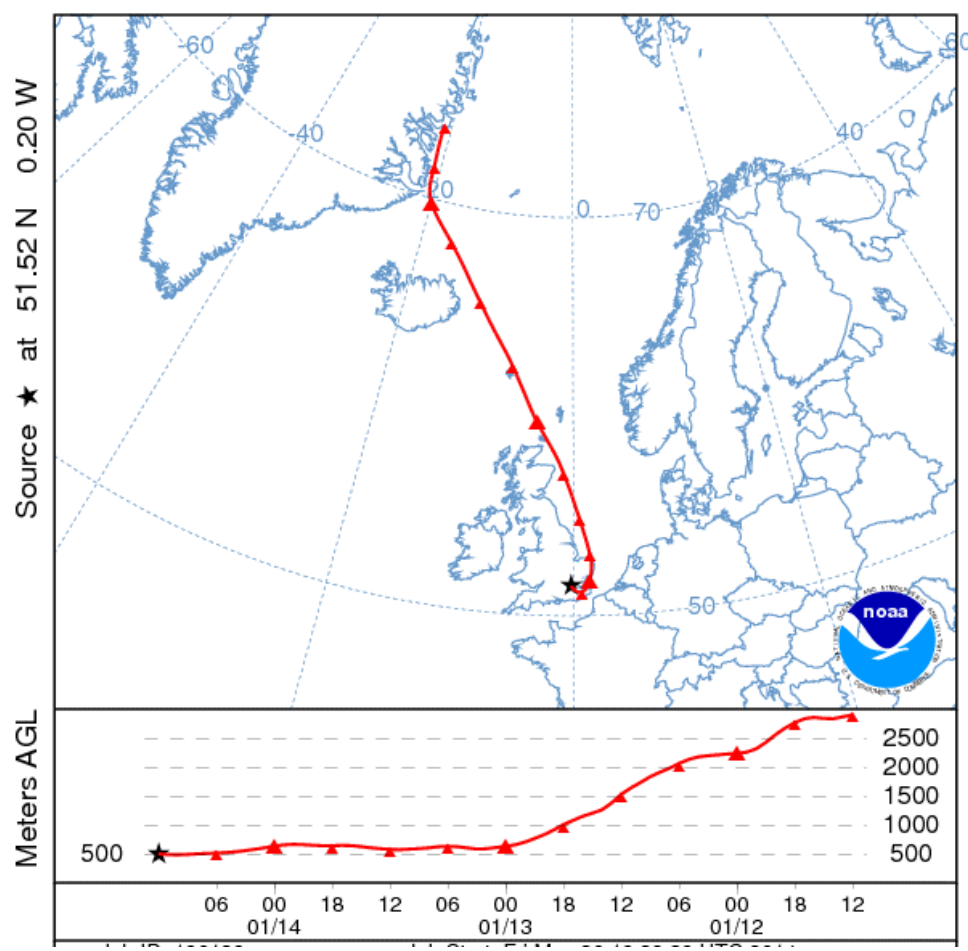

NOAA HYSPLIT MODEL

Backward trajectory ending at 1200 UTC 17 Jan 12 GDAS Meteorological Data

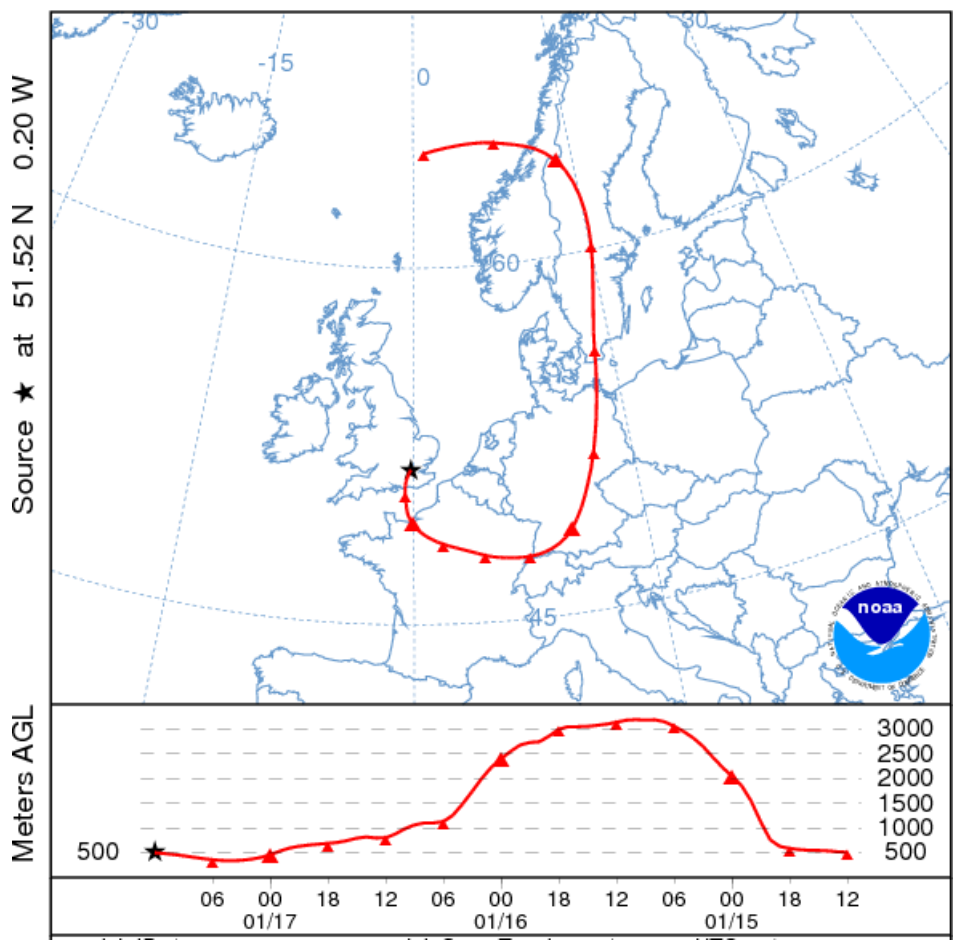


NOAA HYSPLIT MODEL

Backward trajectory ending at 1800 UTC 17 Jan 12

GDAS Meteorological Data

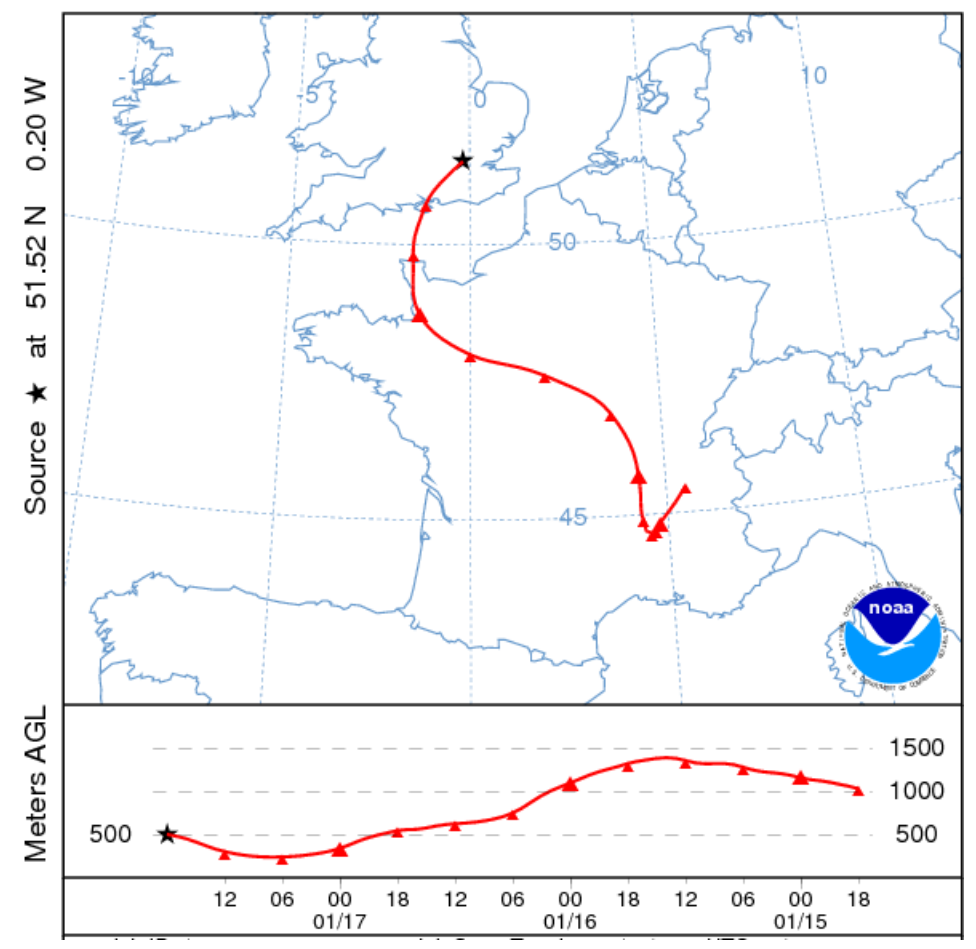

NOAA HYSPLIT MODEL

Backward trajectory ending at 0000 UTC 18 Jan 12 GDAS Meteorological Data

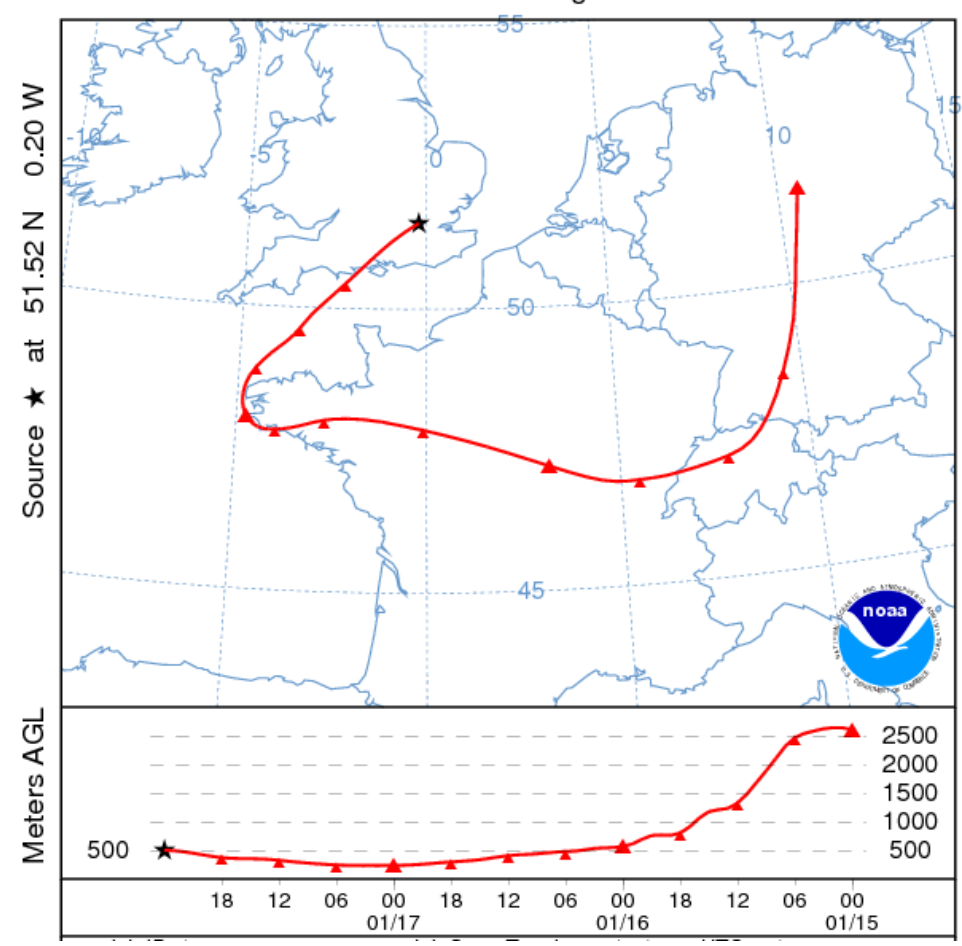


NOAA HYSPLIT MODEL

Backward trajectory ending at 0600 UTC 18 Jan 12

GDAS Meteorological Data

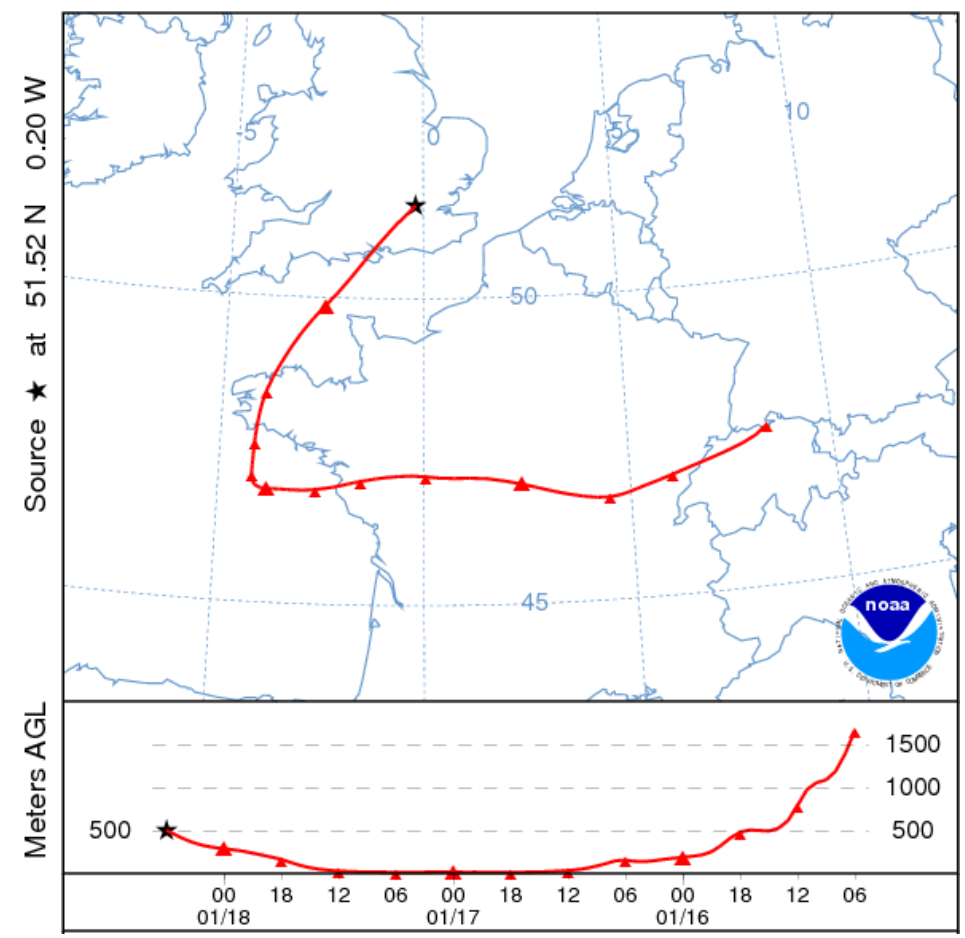

NOAA HYSPLIT MODEL

Backward trajectory ending at 1200 UTC 18 Jan 12 GDAS Meteorological Data

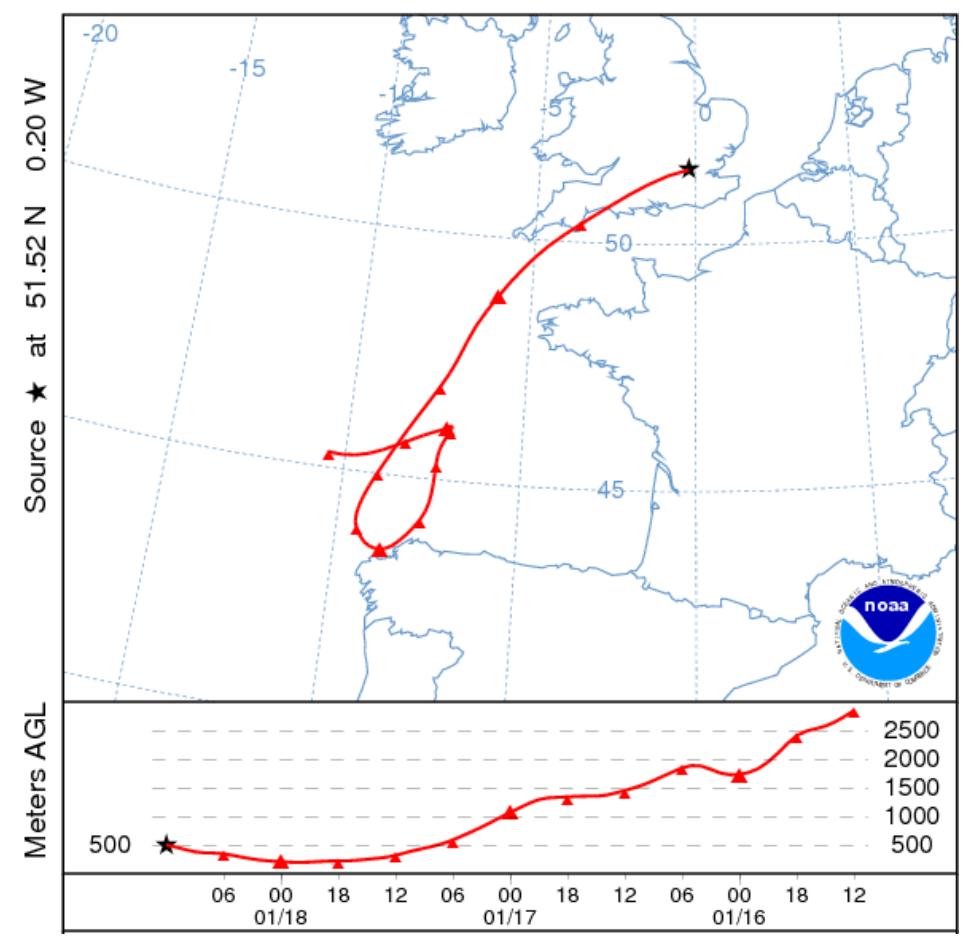


NOAA HYSPLIT MODEL

Backward trajectory ending at 1200 UTC 30 Jan 12

GDAS Meteorological Data

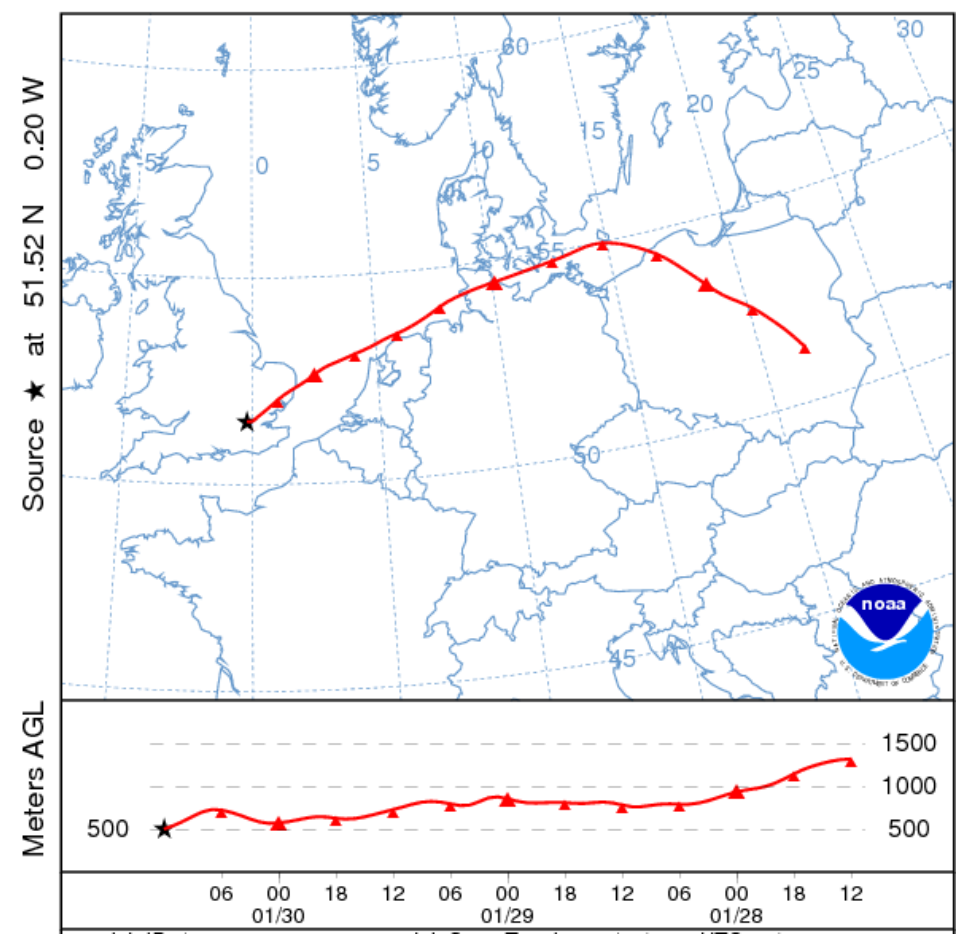

NOAA HYSPLIT MODEL

Backward trajectory ending at 1800 UTC 30 Jan 12

GDAS Meteorological Data

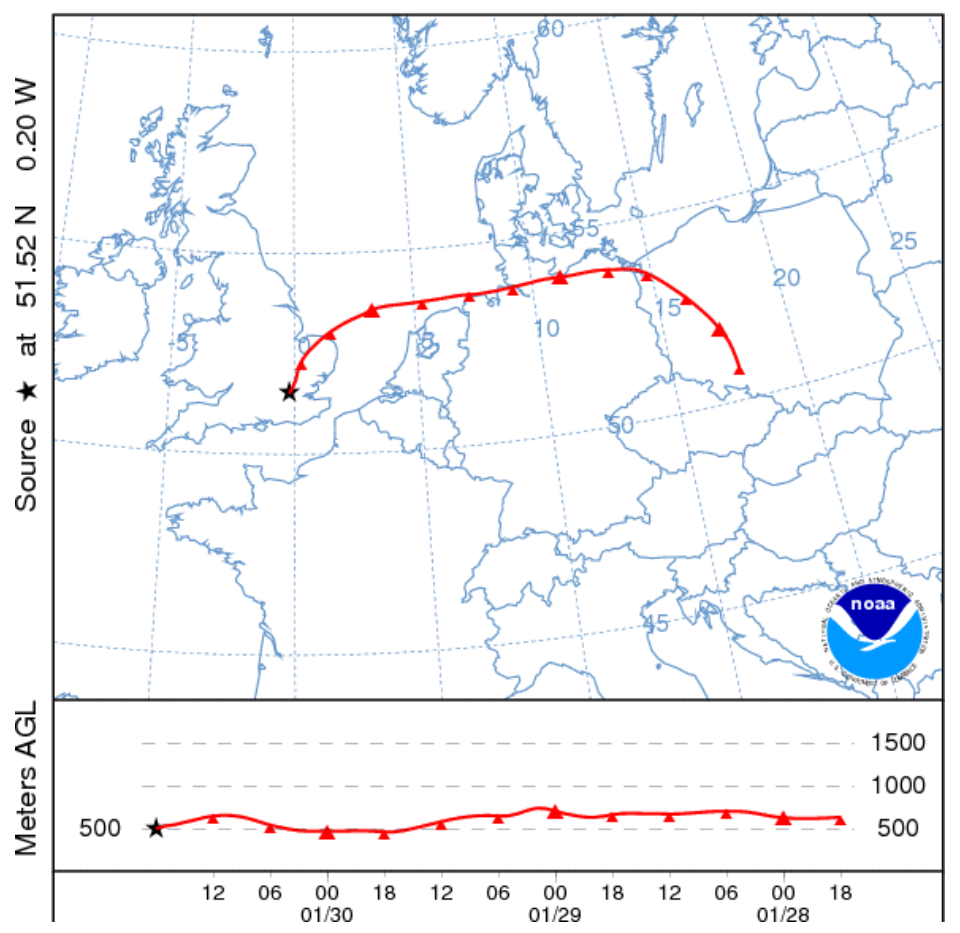


NOAA HYSPLIT MODEL

Backward trajectory ending at 0000 UTC 31 Jan 12

GDAS Meteorological Data

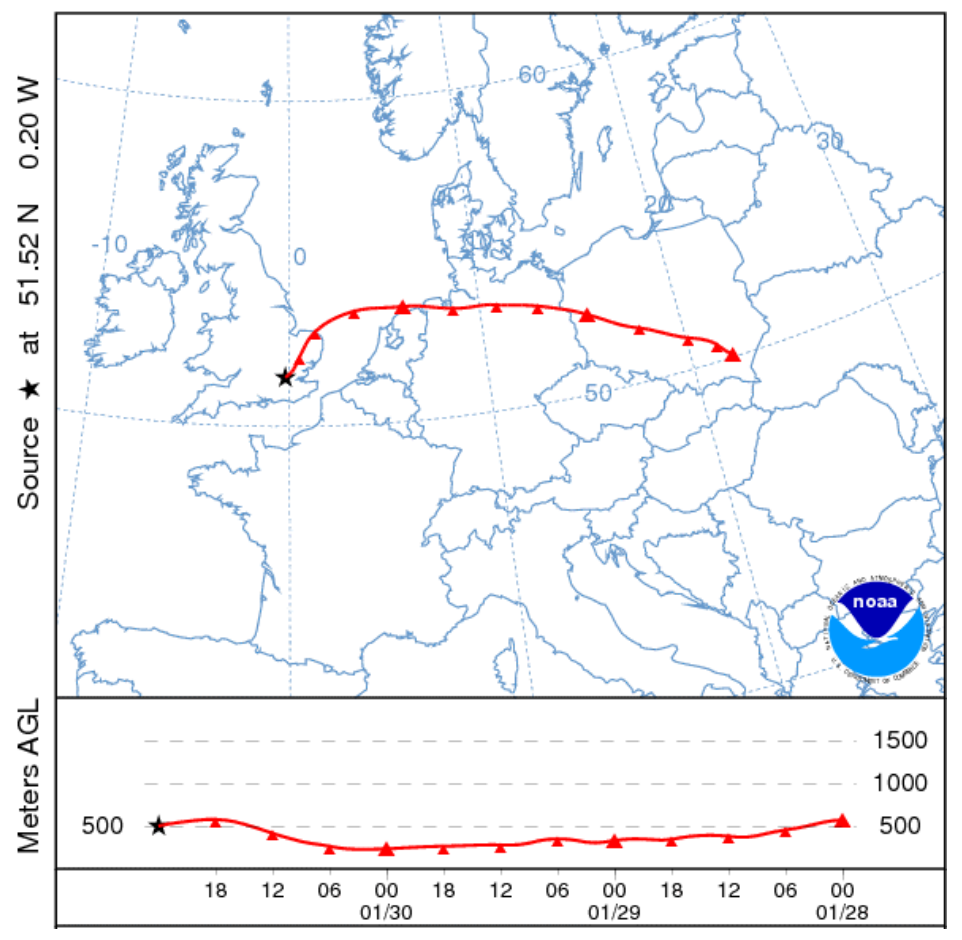

NOAA HYSPLIT MODEL

Backward trajectory ending at 0600 UTC 31 Jan 12 GDAS Meteorological Data

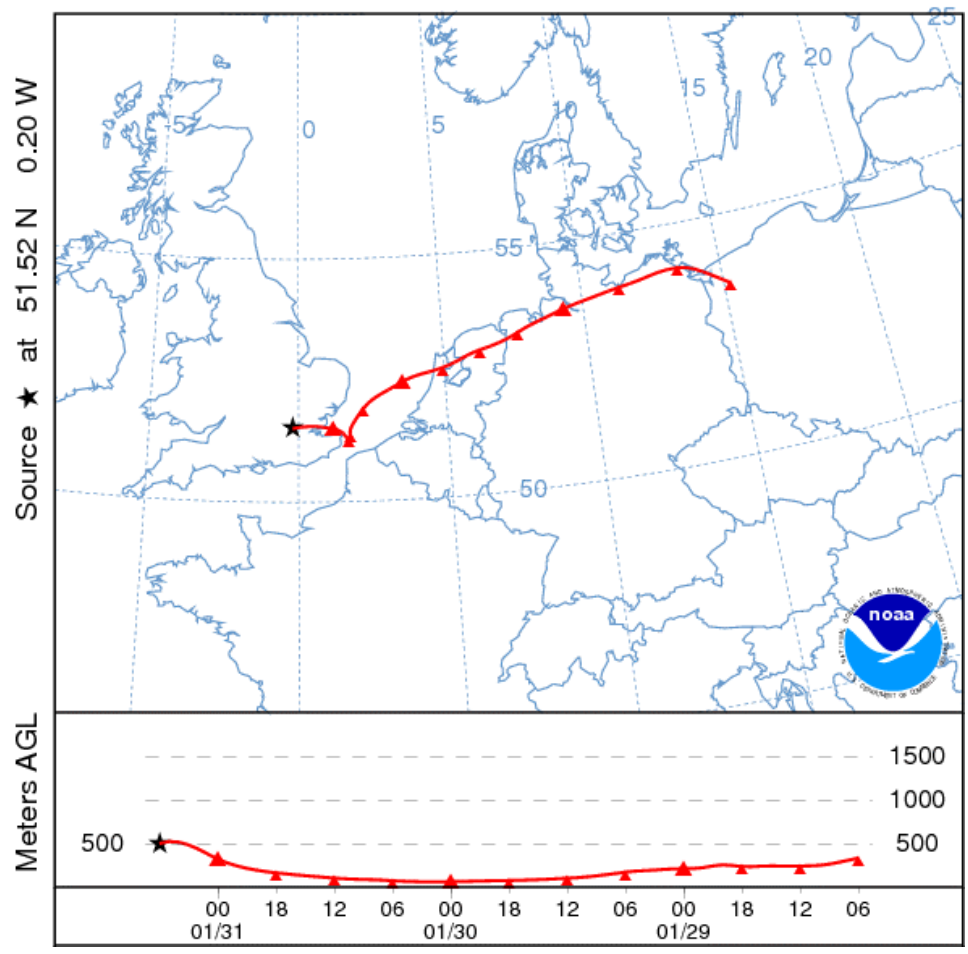


NOAA HYSPLIT MODEL

Backward trajectory ending at 1200 UTC 31 Jan 12

GDAS Meteorological Data

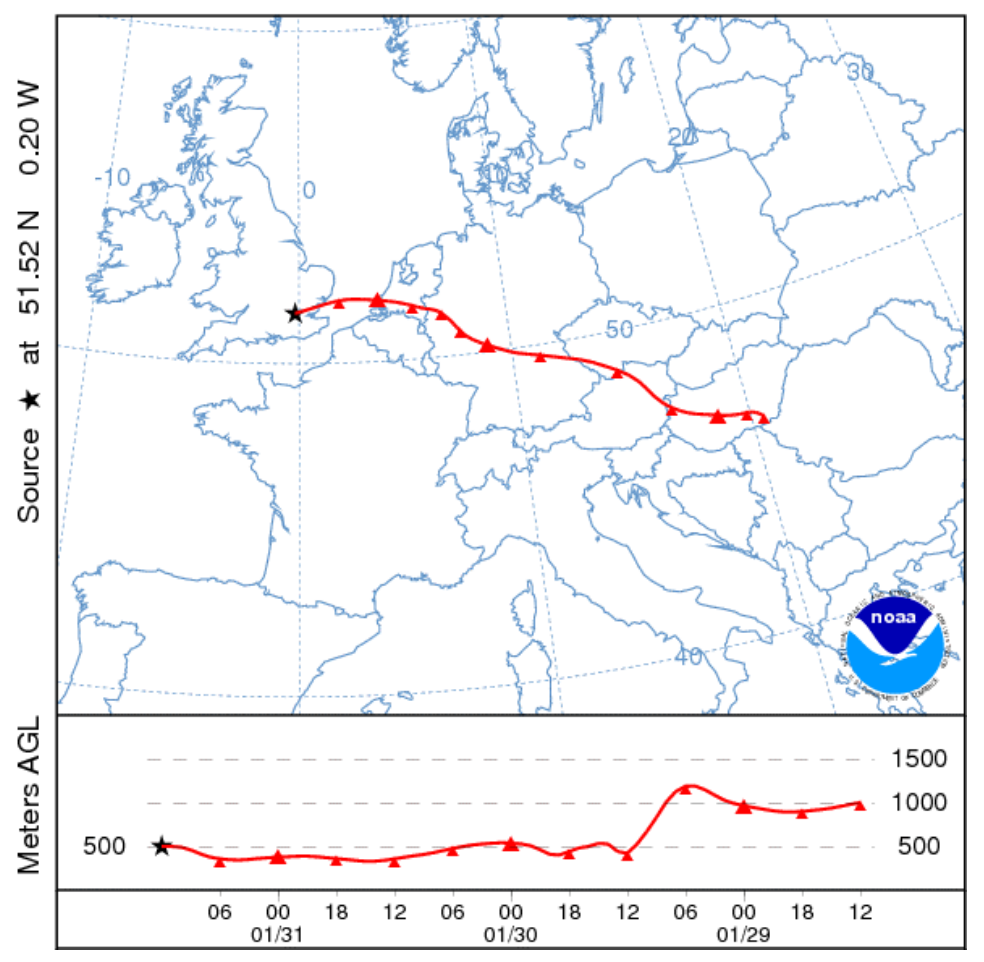

NOAA HYSPLIT MODEL

Backward trajectory ending at 1800 UTC 31 Jan 12 GDAS Meteorological Data

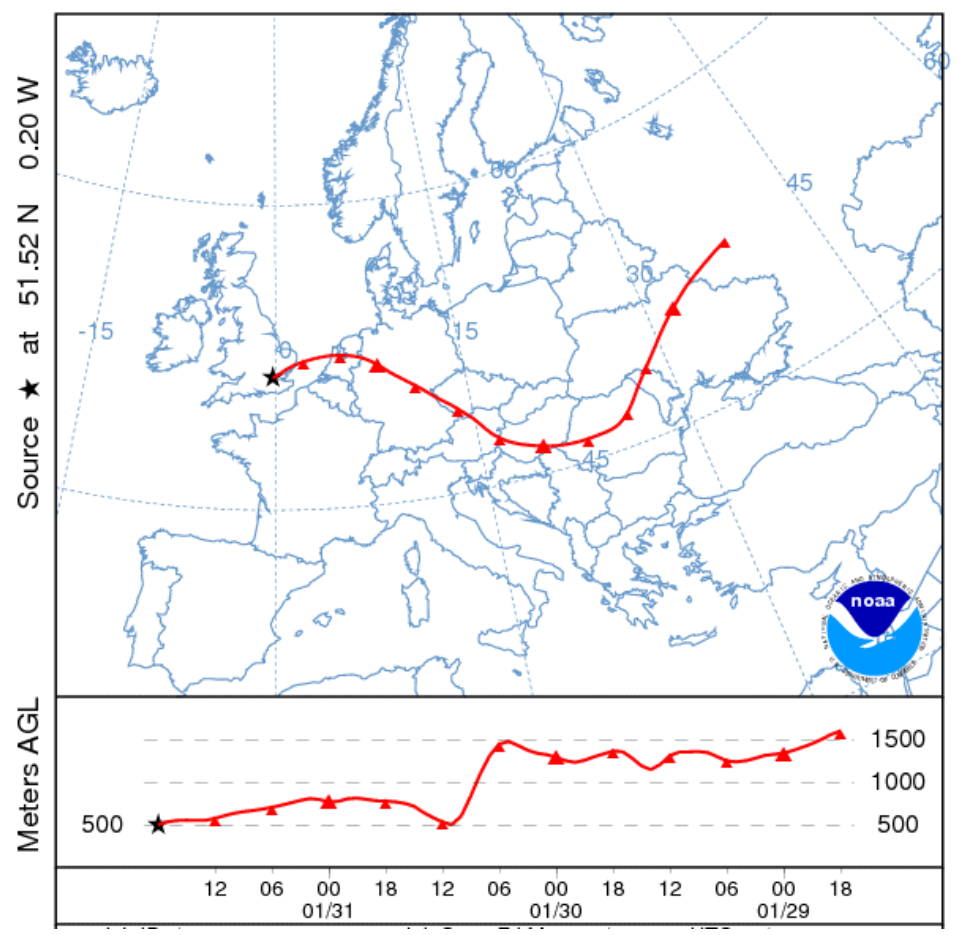


NOAA HYSPLIT MODEL

Backward trajectory ending at 0000 UTC 01 Feb 12

GDAS Meteorological Data

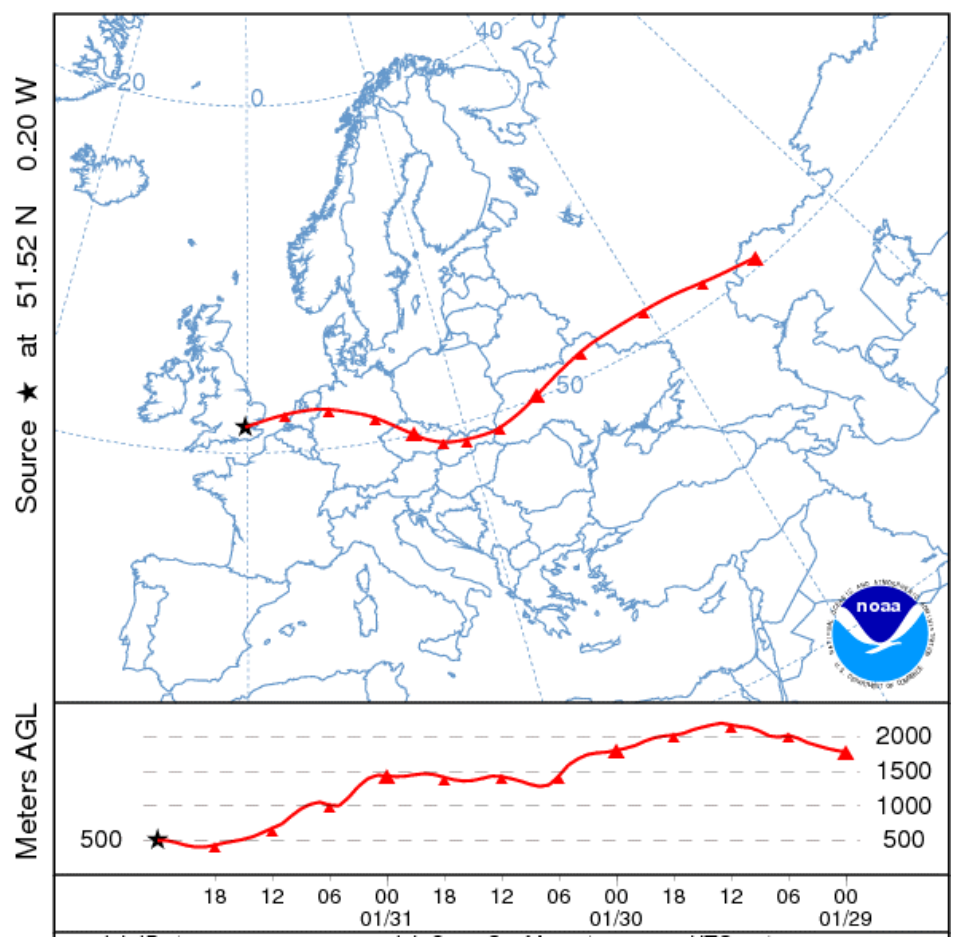

NOAA HYSPLIT MODEL

Backward trajectory ending at 0600 UTC 01 Feb 12 GDAS Meteorological Data

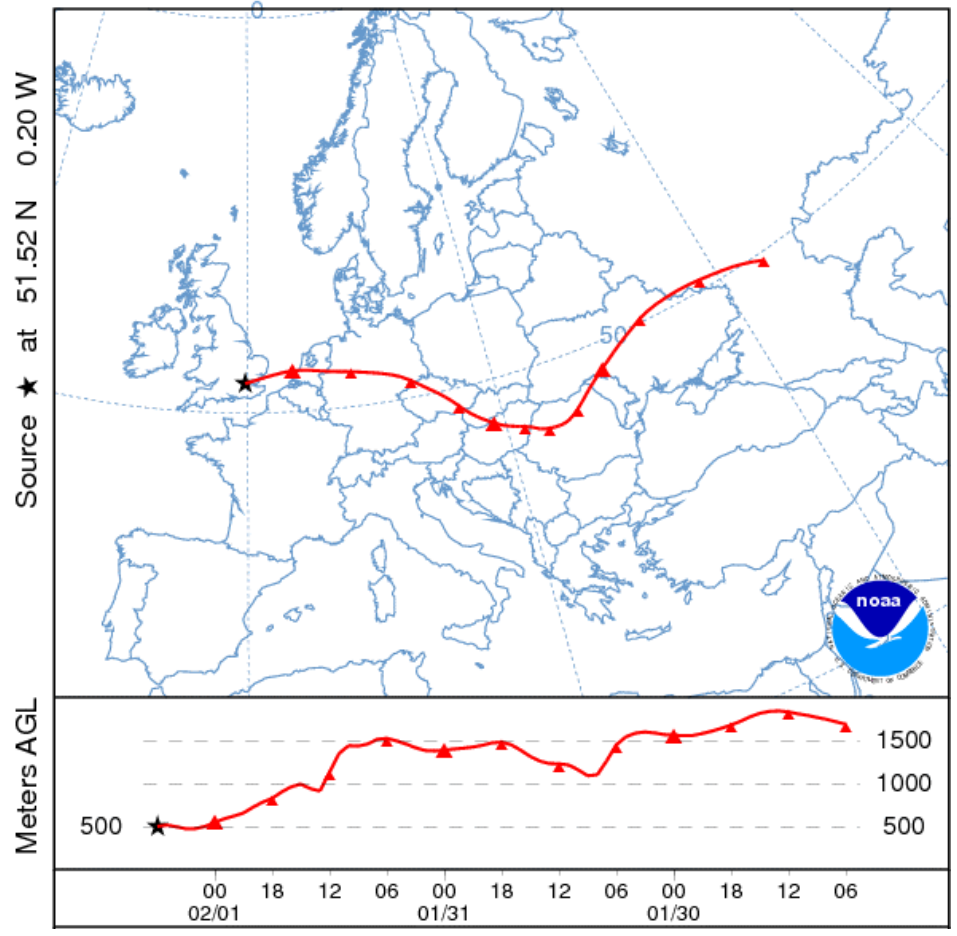


NOAA HYSPLIT MODEL

Backward trajectory ending at 1200 UTC 01 Feb 12

GDAS Meteorological Data

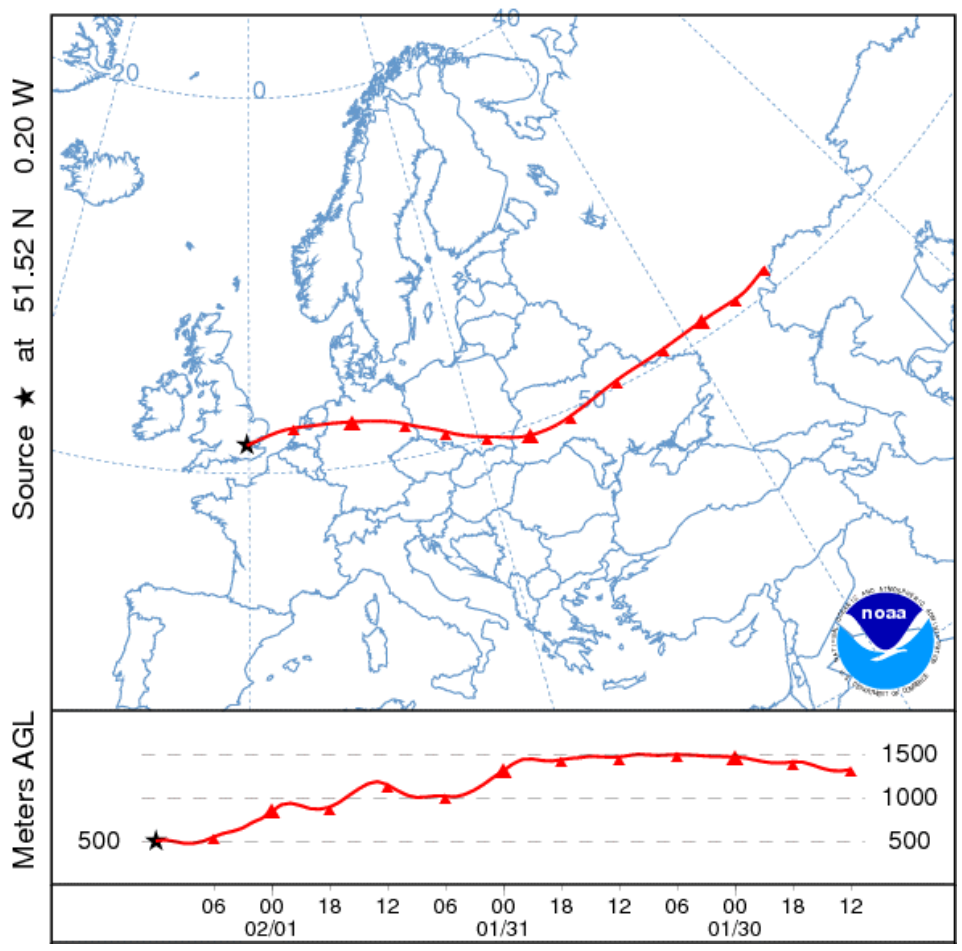

NOAA HYSPLIT MODEL

Backward trajectory ending at 1200 UTC 03 Feb 12 GDAS Meteorological Data

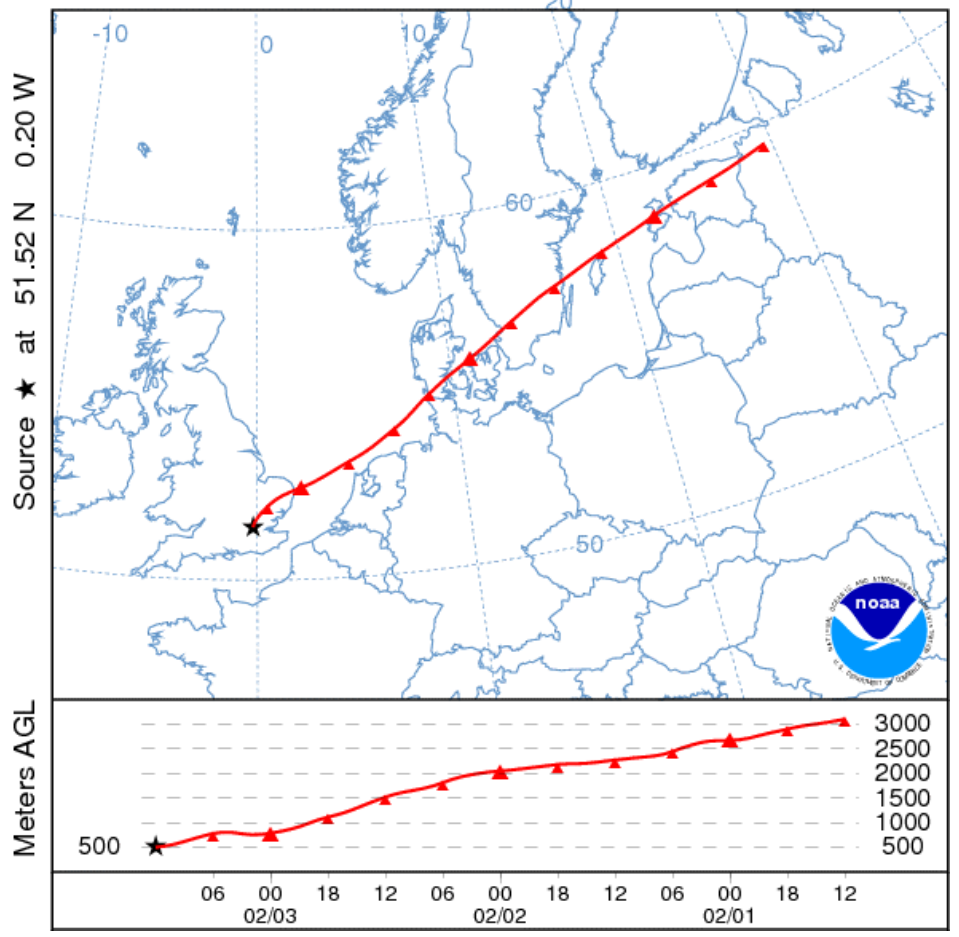


NOAA HYSPLIT MODEL

Backward trajectory ending at 1800 UTC 03 Feb 12

GDAS Meteorological Data

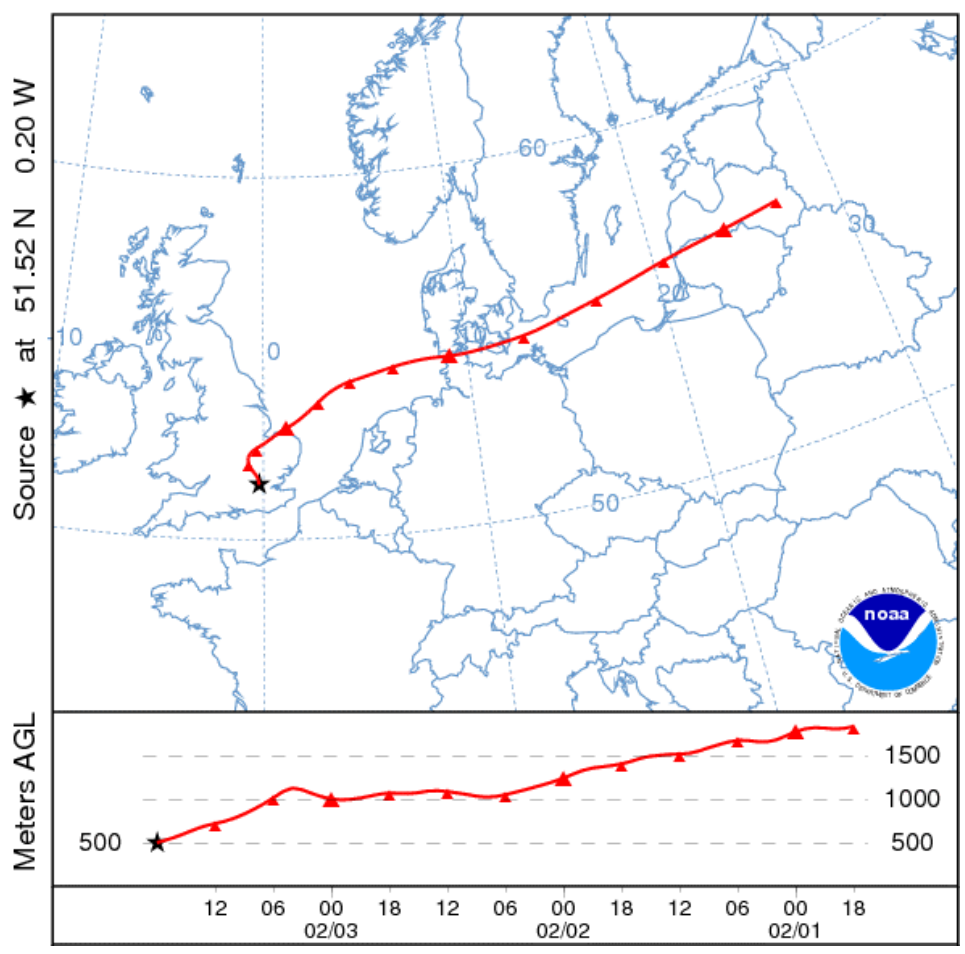

NOAA HYSPLIT MODEL

Backward trajectory ending at 0000 UTC 04 Feb 12

GDAS Meteorological Data

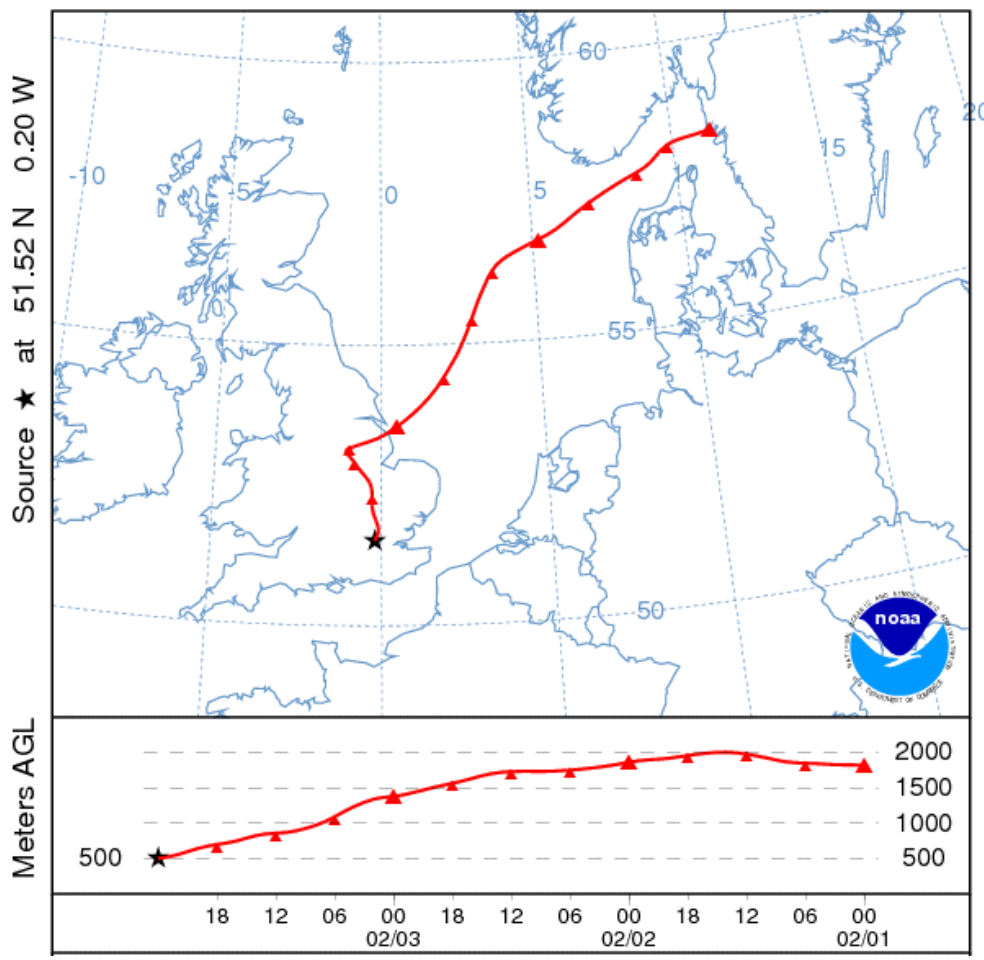


NOAA HYSPLIT MODEL

Backward trajectory ending at 0600 UTC 04 Feb 12

GDAS Meteorological Data

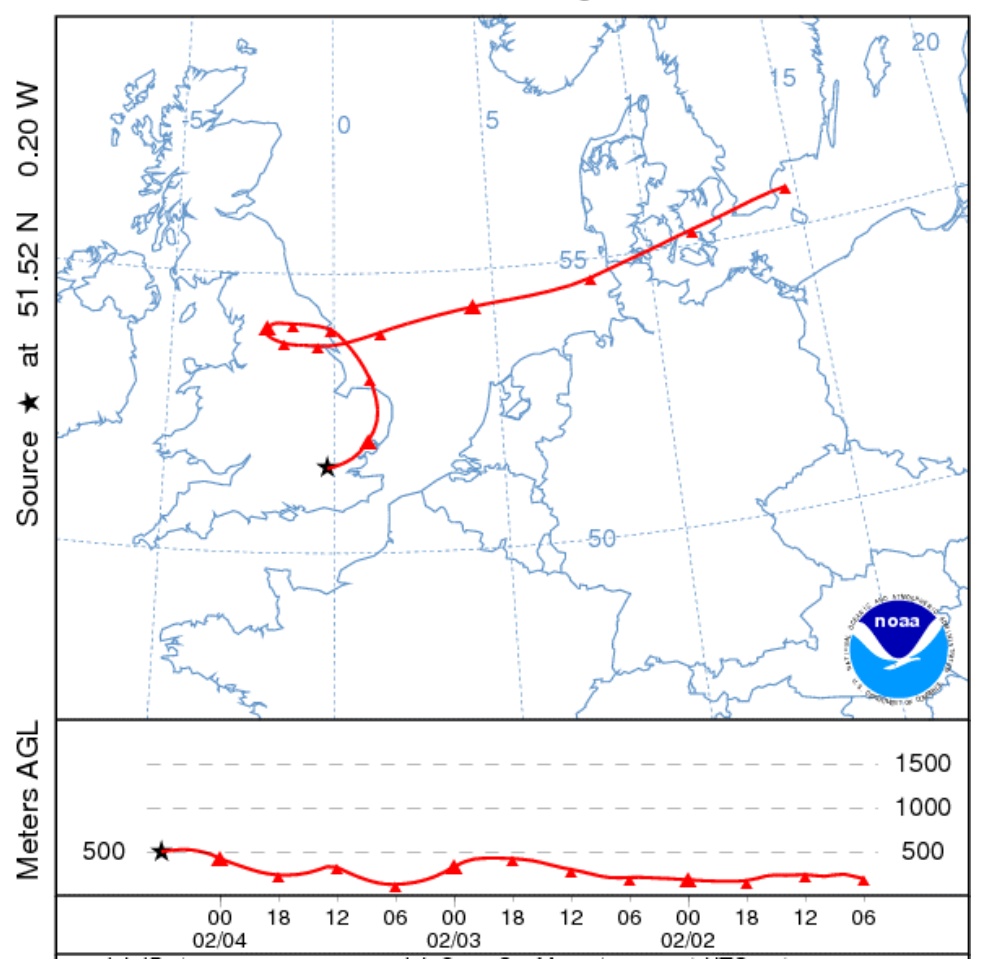

NOAA HYSPLIT MODEL

Backward trajectory ending at 1200 UTC 04 Feb 12 GDAS Meteorological Data

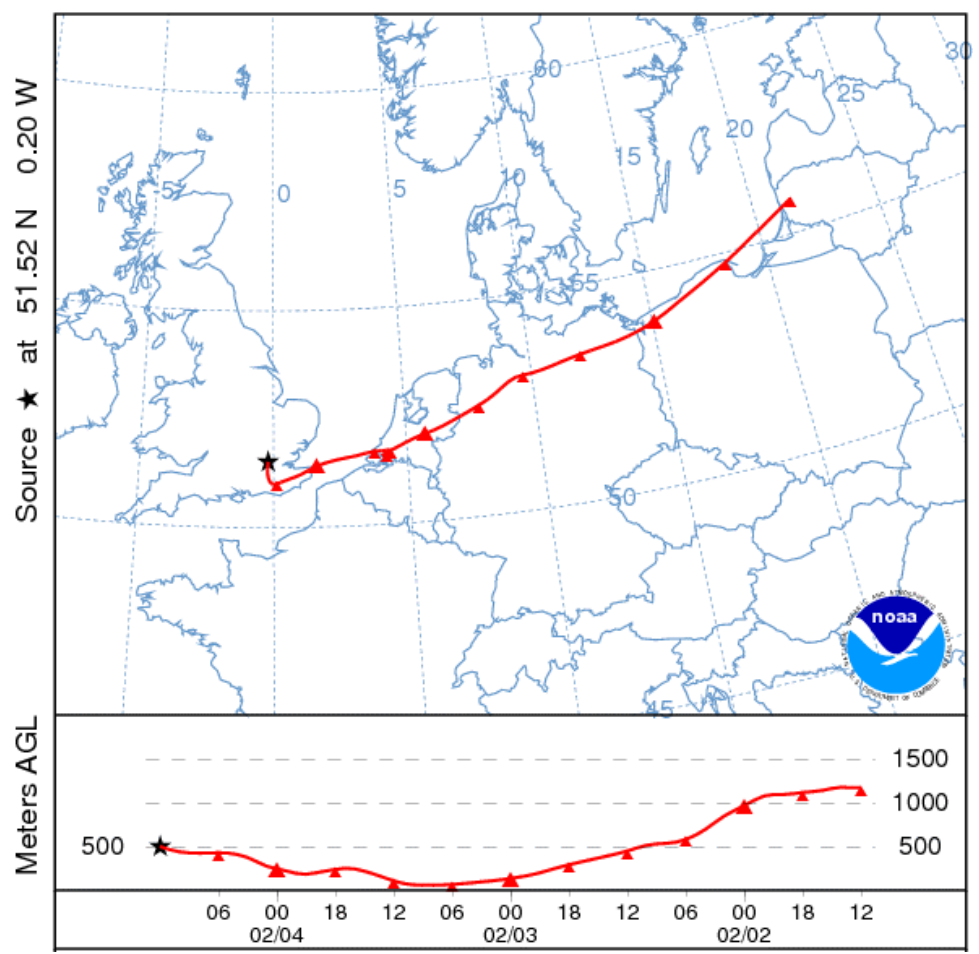


NOAA HYSPLIT MODEL

Backward trajectory ending at 1800 UTC 04 Feb 12

GDAS Meteorological Data

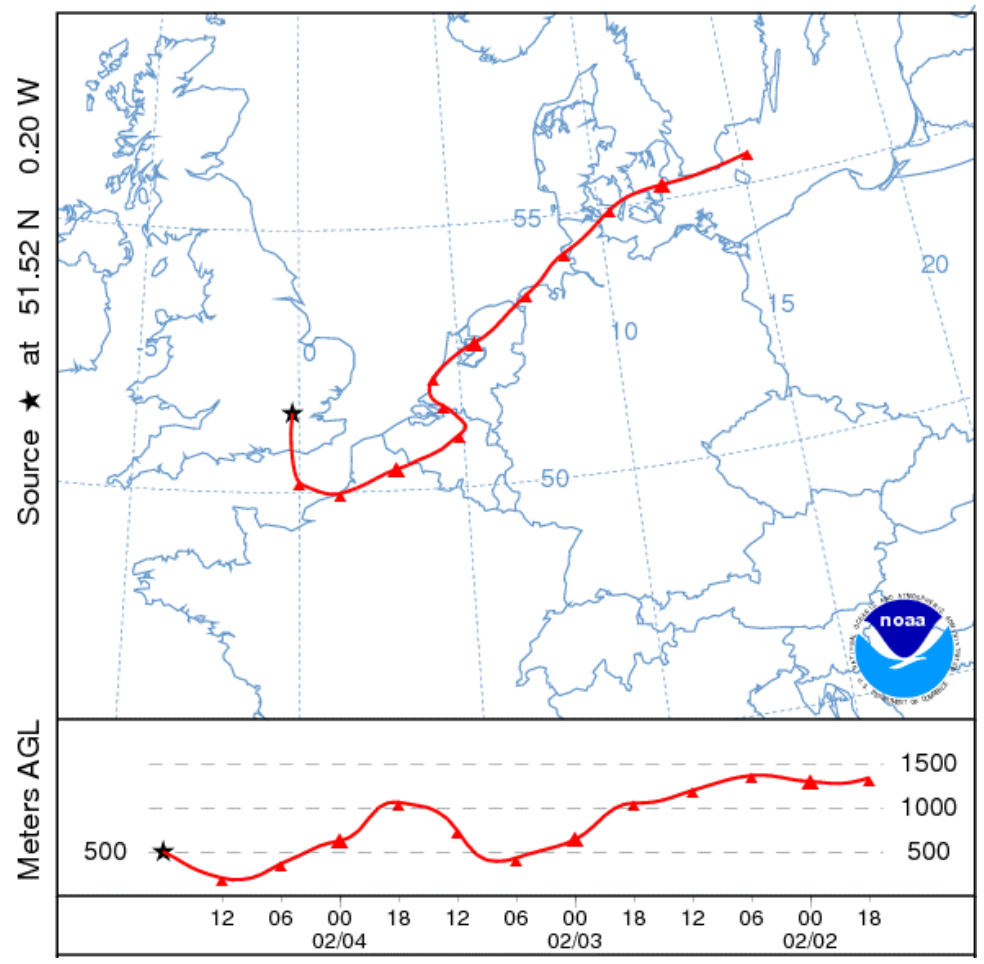

NOAA HYSPLIT MODEL

Backward trajectory ending at 0000 UTC 05 Feb 12 GDAS Meteorological Data

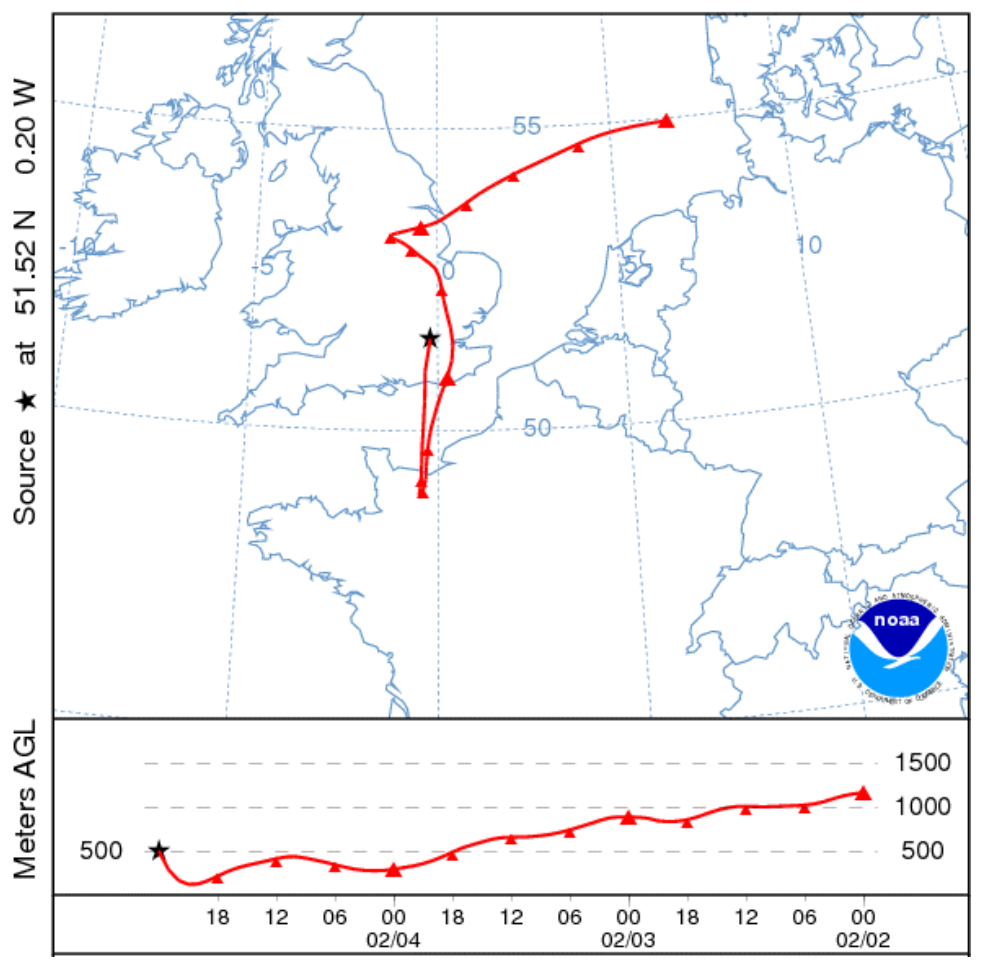


NOAA HYSPLIT MODEL

Backward trajectory ending at 0600 UTC 05 Feb 12

GDAS Meteorological Data

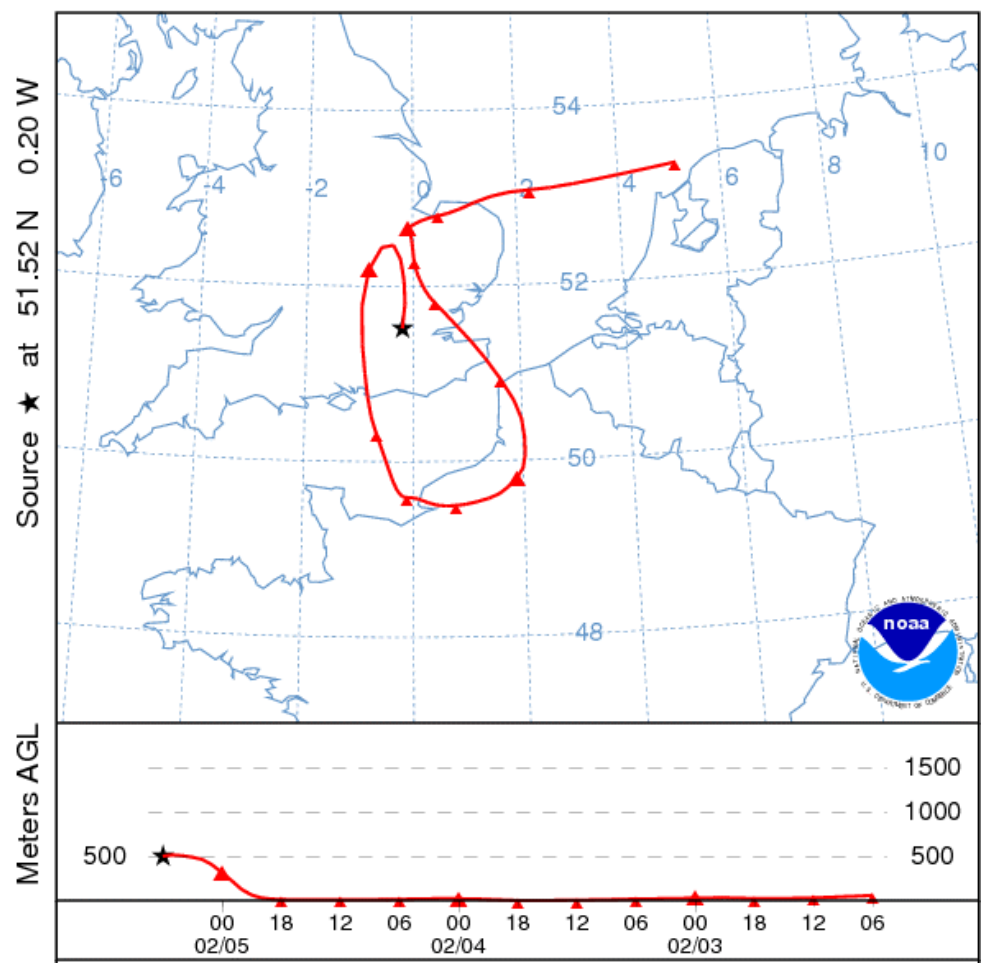

NOAA HYSPLIT MODEL

Backward trajectory ending at 1200 UTC 05 Feb 12 GDAS Meteorological Data

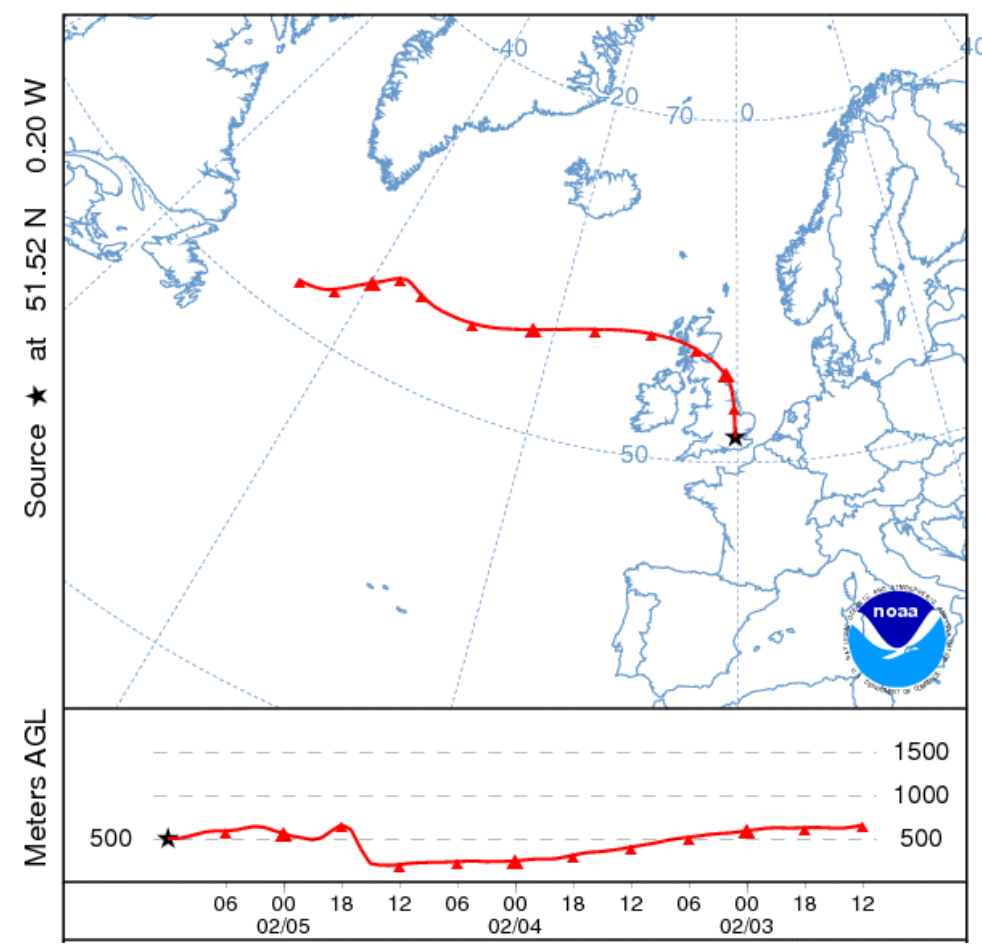

Figure S2. Air mass back trajectories at NK for starting dates of January $13^{\text {th }}, 17^{\text {th }}, 30^{\text {th }}, 31^{\text {st }}$ and February $3^{\text {rd }}, 4^{\text {th }}$. 